\title{
Proceeding
}

Supplementary Issue: Winter Conferences of Sports Science. VI International Congress and XXVII National Congress of Physical

Education, 23-25 October 2019. Palma (Baleares), Spain.

\section{Associations between health-related physical fitness and self-esteem in primary school children}

\author{
ÁLVARO INFANTES-PANIAGUA, JUAN GREGORIO FERNÁNDEZ-BUSTOS , MARÍA PILAR LEÓN, \\ DAVID ZAMORANO-GARCÍA
}

Faculty of Education of Albacete. University of Castilla-La Mancha, Spain

\begin{abstract}
Physical fitness plays an important role as an indicator of physical health. It is also associated with psychological well-being by showing positive relationships between its components and self-esteem in children and adolescents. However, while the musculoskeletal fitness shows clearer associations, this is not the case of cardiorespiratory fitness and body composition. This study aimed to determine the relationships between the different components of the physical fitness and self-esteem in a sample of 377 students from 9 to 13 years in Albacete. Physical fitness was measured by the $20 \mathrm{~m}$ shuttle run, broad jump test and handgrip strength. Waist circumference and body mass index were used as body composition measures. Self-esteem was evaluated with the Multimedia and Multilingual Questionnaire for Self-Esteem Assessment, attending to its academic, athletic, socio-affective and global domains. Results showed significant positive associations of $20 \mathrm{~m}$ shuttle run and broad jump test with global self-esteem and most of the dimensions. There were also significant correlations between body composition measures and athletic and global selfesteem. These results allow for guiding those interventions aimed at increasing self-esteem in school age and incorporating the improvement of physical fitness.

Keywords: Cardiorespiratory fitness; Musculoskeletal fitness; Body weight; Self-esteem; Self-concept; Childhood.

\section{Cite this article as:}

Infantes-Paniagua, A., Fernández-Bustos, J.G., León, M.P., \& Zamorano-García, D. (2020). Associations between health-related physical fitness and self-esteem in primary school children. Journal of Human Sport and Exercise, 15(2proc), S315-S326. doi:https://doi.org/10.14198/ihse.2020.15.Proc2.23

Corresponding author. Faculty of Education of Albacete. University of Castilla-La Mancha, Plaza de la Universidad, 3 (Albacete). 02071. Spain. https://orcid.org/0000-0001-5429-9077

E-mail: JuanG.Fernandez@uclm.es

Supplementary Issue: Winter Conferences of Sports Science. VI International Congress and XXVII National Congress of Physical Education, 23-25 October 2019. Palma (Baleares), Spain.

JOURNAL OF HUMAN SPORT \& EXERCISE ISSN 1988-5202

(c) Faculty of Education. University of Alicante

doi:10.14198/jhse.2020.15.Proc2.23
\end{abstract}




\section{INTRODUCTION}

Overweight and obesity prevalence among European children and adolescents is still an alarming health issue, although its increase seems to have become steady in last years (Garrido-Miguel et al., 2019). The World Health Organization $(2016,2017)$ reports that obesity is the cause of death of more than two and a half million of people every year. This fact highlights the importance of healthy habits (i.e., diet and physical activity) in maintaining a healthy body. In this sense, physical fitness (PF), along with habitual physical activity, plays an important role as a physical health indicator and a predictor of mortality because of cardiovascular risk (DeFina et al., 2015; Ortega et al., 2012). Its role becomes even more relevant when considering that around $20 \%$ of the European children and adolescents are at cardiovascular risk, with a higher prevalence in the southern countries (Tomkinson et al., 2018).

Health-related physical fitness (HPF) is defined as the set of components that an individual owns, which allows them to engage in daily physical activity with vigour, decreasing the risk of chronic diseases or premature death (Bouchard et al., 1994). There are mainly four components: body composition, cardiorespiratory fitness (CRF), musculoskeletal fitness, and motor skills (i.e., speed, agility, and coordination) (Ruiz et al., 2011). In addition to the aforementioned benefits on physical health, HPF, especially CRF, has been associated with a better health-related quality of life in children (Borras et al., 2011). Also, PF plays a moderating role in the relationship between the recent events of life in 6 - to 8-yearsold children and their psychological wellbeing (Gerber et al., 2017), showing that individuals with higher CRF levels presented a lower risk of psychological wellbeing decrease before critical events.

Apart from the quality of life, self-concept is one of the main indicators of psychological wellbeing, especially during childhood and within the academic context (Rosenberg, 1985). Self-esteem is a construct closely related to self-concept. Theoretically, self-concept is defined as the perception that an individual has about themselves, while self-esteem is the assessment that an individual makes on that perception (Shavelson et al., 1976). Nevertheless, such difference has not been empirically demonstrated and, therefore, in practical research self-concept and self-esteem are used interchangeably (Ramos, 2008; Shavelson et al., 1976). Self-concept is a multidimensional, hierarchically-organised construct in which different, more concrete selfconcepts can be distinguished, such as academic, social or physical (Shavelson et al., 1976).

Regarding the association between healthy habits and psychological wellbeing, many studies report positive associations between physical activity and physical self-concept (Babic et al., 2014). Nevertheless, within the PF area, results are mixed: while the positive association between muscular strength and various physical self-perceptions, as well as global self-esteem, is increasingly evident (Smith et al., 2014), the association between CRF and physical and global self-esteem does not seem so clear (Lang et al., 2018). Within the Spanish child population, there is recent evidence showing that those individuals with healthy PF scored higher on physical and social self-concept (Rosa et al., 2019); moreover, individuals who were also active showed better intellectual, social and global self-concepts. Similarly, 8- to 12-years-old students with normal weight and high CRF significantly outscored their peers with lower CRF on physical, behavioural, lack of anxiety, and global self-esteem, regardless their weight status (Gálvez, Rodríguez, Rosa, García-Cantó, Pérez, et al., 2015).

Focusing on children and adolescents' CRF, studies have shown positive associations with life satisfaction (Padilla-Moledo, Castro-Piñero, et al., 2012) and self-esteem (Gálvez, Rodríguez, Rosa, García-Cantó, Pérez, et al., 2015; Grao-Cruces et al., 2017), although other studies did not confirm such associations (Delgado-Floody et al., 2017; Guillén and Ramírez, 2011). Despite the possible lack of associations, 
intervention-based evidence shows a positive effect of endurance on self-esteem (Collins et al., 2019). An increase of various dimensions of self-concept (i.e. endurance, activity level, athletic ability, physical appearance, and global physical self-concepts) has also been demonstrated in obese individuals after a training programme (Rey et al., 2017).

Furthermore, children and adolescents with below-average musculoskeletal fitness were more likely to show lower life satisfaction (Padilla-Moledo, Ruiz et al., 2012). In line with this, Grao-Cruces et al. (2017) found a positive association between handgrip strength and physical and strength self-perceived competences, as well as with self-esteem. They also found an additional positive association with PF self-concept only among males and another negative association with self-perceived appearance among females. No significant associations have also been reported (Delgado-Floody et al., 2017; Guillén and Ramírez, 2011), as well as similar positive associations in overweight and obese students (Rodriguez-Ayllon et al., 2018).

Regarding the third component of HPF, weight status has been highlighted by some studies as a variable related to psychological wellbeing in children and adolescents. Body mass index (BMI) has been negatively associated with physical self-concept and social self-perceived competence (Hill, 2017). Some research found that normal-weight individuals showed higher scores on behavioural, intellectual, physical, and global self-concepts (Gálvez, Rodríguez, Rosa, García-Cantó, Pérez-Soto, et al., 2015; Gálvez, Rodríguez, Rosa, Garcia-Cantó, Pérez, et al., 2015). Other studies have also reported a negative association between BMI and global self-esteem (Delgado-Floody et al., 2017; Grao-Cruces et al., 2017), as well as physical selfconcept subdimensions such as perceived appearance, athletic ability, and PF (Grao-Cruces et al., 2017).

Although results on associations between physical health and psychological wellbeing show overall positive associations with HPF, some evidence remains unclear. This highlights the need for future studies to analyse these associations in more detail (Rosa-Guillamón, 2019). It is important to differentiate which HPF components may be strongly associated with different aspects of psychological health (Rodriguez-Ayllon et al., 2018). Therefore, the main aim of the present study was to determine the associations between the different components of HPF and self-esteem in a sample of children and young adolescents.

\section{MATERIALS AND METHODS}

\section{Participants}

Three hundred and seventy-seven students (199 females and 178 males) from 9 to 13 years old ( $M=10.49$; $S D=0.93)$ participated in this cross-sectional study. They were enrolled in the fourth, fifth, and sixth year of Primary Education of four different schools in Albacete (Spain). The sample was selected by convenience and parents or legal guardians gave their informed consent for their children to participate. After data inspection, 21 participants were deleted due to mistakes on data gathering or coding in the self-esteem measures; therefore, the final sample was composed of 356 participants.

\section{Measures}

Three components of HPF were measured: CRF, musculoskeletal fitness, and body composition. To assess the first component, the $20 \mathrm{~m}$ shuttle-run test (20mSRT; Léger et al., 1988) was employed. The last stage completed was used as reference. Musculoskeletal fitness was measured through the standing broad jump (SBJ) and the handgrip strength (HS) tests, using a dynamometer Carmy model EH101. Body composition was measured through the waist circumference (WC) and BMI. The height and weight were measured using a stadiometer (Tanita HR-001) and a digital scale (Tanita HD-366), respectively. 
To classify and analyse the participants' results on 20mSRT, SBJ and HS tests, the normative values from the European population were used (Tomkinson et al., 2018), according to sex and age. The resultant percentiles were reorganised by following the categorisation proposed by Ortega et al. (2011): very poor (< P20), poor ( $\geq$ P20 to $<$ P40), medium ( $\geq$ P40 to $<$ P60), good ( $\geq$ P60 to < P80) and very good $(\geq P 80)$. BMl was categorised according to the cut-off points of the International Obesity Task of Force (IOTF; Cole and Lobstein, 2012), and then, grouped into the four main categories of underweight, normal weight, overweight, and obesity. Finally, to classify WC, the percentile reference values of a Spanish sample were used (Fernández et al., 2011).

Participants' self-esteem was assessed using the Spanish version of the Self-esteem Multimedia and Multilingual Evaluation (Cuestionario Multimedia y Multilingüe de Evaluación de la Autoestima, CMMEA; Ramos, 2008). This questionnaire is aimed at a child population from 9 to 13 years. It is composed of 30 items and the answers are based on a three-point Likert scale ('Always', 'Sometimes', 'Never'). The CMMEA has shown good psychometric properties (Ramos, 2008). This instrument is based on the multidimensional model of self-concept proposed by Shavelson et al. (1976). It includes three scales addressing academic self-esteem (AcSE; 9 items, e.g. 'I am happy with my marks'), athletic self-concept (AtSE; 4 items, e.g., 'I'm awkward for most sports'), and socio-affective and emotional self-esteem (SSE; 17 items, e.g. 'I'm happy'). The sum of these three scales provides a score of global self-esteem (GSE). The scores were recoded according to the percentile values by age and sex reported by Ramos (2008).

\section{Procedures}

First, teachers and school principals were contacted and, once they allowed the research team to conduct the study, the participants' parents or legal guardians were informed about the aims and characteristics of the study. Only those students whose parents or legal guardians had given their written consent participated in the study.

Self-esteem questionnaires were filled during a tutoring session in a space that ensured the privacy of responses. Prior to data collection, researchers gave participants oral guidelines about how to fill the questionnaires and highlighted the importance of honestly answering. Despite there was no time limit, participants did not take more than 20 minutes in filling the questionnaires.

HPF tests were developed during two Physical Education lessons by following the sequencing recommended by Ruiz et al. (2011): 1) Weight and height; 2) WC; 3) HS and SBJ; and 4) 20mSRT. Data collection was conducted by two trained researchers to ensure the standardisation and reliability of measurements. The protocol established for the ALPHA-Fitness Battery (Ruiz et al., 2011) was followed.

The procedures complied with the deontological guidelines of the Declaration of Helsinki (Hong-Kong revision, 1989). This study was approved by the ethics committee of the University of Castilla-La Mancha.

\section{Analysis}

Statistical analyses were run using SPSS 24 software (IBM Corporation, Armonk, NY, USA) on the basis of a significance level of .05. First, Kolmogorov-Smirnov test was used to examine whether variables were normally distributed. Despite this assumption was not met for some variables, the normality was assumed according to the Central Limit Theorem due to the sample size. Outliers were checked by transforming the data into z-values and exploring those lower than -3.29 or higher than 3.29 (Field, 2013). No outliers were found. Second, descriptive analyses were run on means and standard deviations of variables. Also, sexdifferences were checked by t-tests (Table 1). Pearson's bivariate correlations were run (Table 2), showing 
overall small-to-medium scores and, thus, the intercorrelation assumption was discarded. Last, in order to examine the differences in self-esteem according to the HPF, a one-way ANOVA test was run for each selfconcept dimension (Table 3). For those variables reporting significant $F$ scores, Tukey's HSD post hoc tests were run (Tables 4 and 5). In the analyses for 20mSRT the included sample was $\mathrm{N}=353$ (171 males, 182 females); and in the analyses for SBJ, HS, WC and BMI the included sample was $N=355$ (172 males, 183 females).

\section{RESULTS}

Descriptive data of all measured variables are reported in Table 1. Sex differences were found in HS, WC, AcSE and AtSE, with males outscoring females only in the latter variable. Bivariate correlations (Table 2) showed positive associations between 20mSRT and AcSE $(r=.141, p<.01)$, AtSE $(r=.297, p<.001)$, SSE $(r=.117, p<.05)$, and GSE $(r=.220, p<.001)$. Also, lower-body muscular strength was positively associated with all self-esteem dimensions (AcSE: $r=.173, p<.01$; AtSE: $r=.244, p<.001$; SSE: $r=.190, p<.001$; GSE: $r=.248, p<.001$ ); however, upper-body strength was not associated with any self-esteem dimensions. In addition, a negative association between body weight and AtSE was found for both WC $(r=-.141, p<.01)$ and BMI $(r=-.171, p<.01)$.

Table 1. Descriptive data.

\begin{tabular}{|c|c|c|c|c|c|c|c|c|}
\hline \multirow{2}{*}{ Variables } & \multicolumn{2}{|c|}{ Total $(\mathrm{N}=356)$} & \multicolumn{2}{|c|}{ Males $(\mathrm{N}=172)$} & \multicolumn{2}{|c|}{ Females $(\mathrm{N}=184)$} & \multicolumn{2}{|c|}{ Sex differences } \\
\hline & M & SD & $\mathbf{M}$ & SD & M & SD & $t$ & $p$ \\
\hline Age (years) & 10.48 & 0.94 & 10.48 & 0.98 & 10.48 & 0.91 & 0.08 & .945 \\
\hline $20 \mathrm{mSRT}$ a & 31.15 & 27.78 & 31.32 & 27.55 & 30.99 & 28.07 & 0.22 & .912 \\
\hline SBJa & 28.92 & 26.36 & 29.51 & 25.72 & 28.36 & 27.01 & 0.32 & .683 \\
\hline $\mathrm{HS}^{\mathrm{a}}$ & 50.39 & 25.49 & 46.54 & 25.31 & 54.02 & 25.19 & 9.21 & $.006^{* *}$ \\
\hline WCa & 65.39 & 29.54 & 61.28 & 30.77 & 69.26 & 27.87 & 8.25 & $.011^{*}$ \\
\hline BMI b & 19.40 & 3.49 & 19.39 & 3.50 & 19.42 & 3.48 & 0.15 & .944 \\
\hline AcSE b & 13.54 & 2.98 & 13.13 & 3.09 & 13.91 & 2.83 & 6.61 & $.013^{*}$ \\
\hline AtSE b & 6.74 & 1.95 & 7.08 & 1.68 & 6.43 & 2.12 & 10.64 & $.002^{* *}$ \\
\hline SSE b & 28.77 & 4.65 & 28.67 & 4.77 & 28.86 & 4.54 & 0.14 & .701 \\
\hline GSE b & 49.05 & 7.19 & 48.89 & 7.25 & 49.21 & 7.15 & 0.17 & .678 \\
\hline
\end{tabular}

a percentile values; ${ }^{b}$ absolute values. ${ }^{*} p<.05 ;{ }^{* *} p<.01$.

M: mean, SD: standard deviation, t: t-test. 20mSRT: 20m shuttle run test; SBJ: standing broad jump; HS: handgrip strength; WC: waist circumference; BMI: body mass index; AcSE: academic self-esteem; AtSE: athletic self-esteem; SSE: socio-affective and emotional self-esteem; GSE: global self-esteem.

Table 2. Pearson's bivariate correlations between physical fitness and self-esteem variables (percentiles).

\begin{tabular}{llllc}
\hline & AcSE & AtSE & SSE & GSE \\
\hline 20mSRT & $.141^{* *}$ & $.297^{* * *}$ & $.117^{*}$ & $.220^{\star * *}$ \\
SBJ & $.173^{\star *}$ & $.244^{* * *}$ & $.190^{* * *}$ & $.248^{\star * *}$ \\
HS & .096 & -.015 & .080 & .085 \\
WC & .083 & $-.141^{* *}$ & .045 & .020 \\
BMI & -.001 & $-.171^{* *}$ & .017 & -.037 \\
\hline
\end{tabular}

${ }^{*} p<.05,{ }^{* *} p<.01,{ }^{* * *} p<.001$.

20mSRT: $20 \mathrm{~m}$ shuttle run test; SBJ: standing broad jump; HS: handgrip strength; WC: waist circumference; BMI: body mass index; AcSE: academic self-esteem; AtSE: athletic self-esteem; SSE: socio-affective and emotional self-esteem; GSE: global self-esteem. 
Table 3. One-way ANOVA tests for each self-esteem dimension.

\begin{tabular}{lcccccccc}
\hline \multirow{2}{*}{ HPF } & \multicolumn{2}{c}{ AcSE } & \multicolumn{2}{c}{ AtSE } & \multicolumn{2}{c}{ SSE } & \multicolumn{2}{c}{ GSE } \\
\cline { 2 - 9 } & $\mathbf{F}$ & $\mathbf{p}$ & $\mathbf{F}$ & $\mathbf{p}$ & $\mathbf{F}$ & $\mathbf{p}$ & $\mathbf{F}$ & $\mathbf{p}$ \\
\hline 20mSRT & 3.32 & $.011^{*}$ & 8.89 & $.000^{* *}$ & 1.18 & .315 & 4.61 & $.001^{* *}$ \\
SBJ & 3.32 & $.011^{*}$ & 6.78 & $.000^{* *}$ & 4.58 & $.001^{* *}$ & 8.14 & $.000^{* * *}$ \\
HS & 1.08 & .366 & 0.265 & .901 & 1.69 & .152 & 1.62 & .169 \\
WC & 1.12 & .340 & 4.87 & $.002^{* *}$ & 0.46 & .707 & 1.87 & .133 \\
BMI & 1.36 & .254 & 3.76 & $.011^{*}$ & 1.82 & .143 & 3.04 & $.029^{*}$ \\
\hline
\end{tabular}

${ }^{*} p<.05,{ }^{* *} p<.01,{ }^{* * *} p<.001$.

20mSRT: $20 \mathrm{~m}$ shuttle run test; SBJ: standing broad jump; HS: handgrip strength; WC: waist circumference; BMI: body mass index; AcSE: academic self-esteem; AtSE: athletic self-esteem; SSE: socio-affective and emotional self-esteem; GSE: global self-esteem.

Table 4. Means and SD from post hoc tests in 20mSRT and SBJ.

\begin{tabular}{|c|c|c|c|c|c|c|c|}
\hline \multirow{2}{*}{ Self-esteem } & \multirow{2}{*}{ Subgroup } & \multicolumn{3}{|c|}{ 20mSRT } & \multicolumn{3}{|c|}{ SBJ } \\
\hline & & $\mathrm{n}$ & M & SD & $\mathrm{n}$ & $M$ & SD \\
\hline \multirow{6}{*}{ AcSE } & a & 138 & 52.20 & 28.30 & 147 & 49.17 & 27.47 \\
\hline & b & 87 & 49.19 & 29.00 & 81 & 55.49 & 28.48 \\
\hline & c & 51 & 63.60 & 26.79 & 61 & 56.80 & 29.65 \\
\hline & $d$ & 43 & 57.62 & 28.99 & 40 & 65.00 & 25.58 \\
\hline & $\mathrm{e}$ & 34 & 63.05 & 22.18 & 26 & 61.69 & 27.12 \\
\hline & Sig. differences & \multicolumn{3}{|c|}{$b<c$} & \multicolumn{3}{|c|}{$a<d$} \\
\hline \multirow{6}{*}{ AtSE } & $a$ & 138 & 47.51 & 29.40 & 147 & 49.31 & 28.70 \\
\hline & b & 87 & 56.31 & 25.30 & 81 & 58.29 & 24.31 \\
\hline & c & 51 & 61.35 & 23.24 & 61 & 55.96 & 27.09 \\
\hline & $d$ & 43 & 61.46 & 22.66 & 40 & 67.07 & 16.83 \\
\hline & $\mathrm{e}$ & 34 & 73.52 & 11.57 & 26 & 71.11 & 17.47 \\
\hline & Sig. differences & \multicolumn{3}{|c|}{$a<c, d, e, b<e$} & \multicolumn{3}{|c|}{$a<d, e$} \\
\hline \multirow{6}{*}{ SSE } & $a$ & 138 & 53.13 & 29.30 & 147 & 49.34 & 28.00 \\
\hline & b & 87 & 54.81 & 28.03 & 81 & 59.81 & 26.62 \\
\hline & c & 51 & 58.11 & 27.83 & 61 & 57.57 & 30.13 \\
\hline & $d$ & 43 & 58.18 & 25.63 & 40 & 63.95 & 24.23 \\
\hline & $\mathrm{e}$ & 34 & 63.64 & 22.79 & 26 & 67.23 & 22.18 \\
\hline & Sig. differences & & - & & \multicolumn{3}{|c|}{$a<b, d, e$} \\
\hline \multirow{6}{*}{ GSE } & $a$ & 138 & 51.09 & 27.92 & 147 & 48.11 & 26.76 \\
\hline & b & 87 & 54.11 & 28.02 & 81 & 60.48 & 27.30 \\
\hline & c & 51 & 63.33 & 26.01 & 61 & 58.23 & 30.52 \\
\hline & $d$ & 43 & 60.83 & 28.58 & 40 & 70.75 & 23.36 \\
\hline & $\mathrm{e}$ & 34 & 69.91 & 23.55 & 26 & 68.07 & 20.18 \\
\hline & Sig. differences & \multicolumn{3}{|c|}{$a, b<e$} & \multicolumn{3}{|c|}{$a<b, d, e$} \\
\hline
\end{tabular}

M: mean, SD: standard deviation, $n=$ sample size in subgroup.

20mSRT: 20m shuttle run test; SBJ: standing broad jump; AcSC: academic self-esteem; AtSE: athletic self-esteem; SSE: socioaffective and emotional self-esteem; and GSE: global self-esteem.

a: very poor ( $<$ P20), b: poor ( $\geq P 20$ to $<P 40)$, c: medium ( $\geq P 40$ to $<P 60)$, d: good $(\geq P 60$ to $<P 80)$, and e: very good $(\geq P 80)$.

Regarding ANOVA tests (Table 3), there were significant differences in self-esteem between the established subgroups according to CRF, SBJ, WC, and BMI. No differences were found between the subgroups according to HS. In particular, ACSE, AtSE, and GSE reported a significant F statistic on tests regarding 
20mSRT. Significant differences were observed in all self-esteem dimensions according to SBJ's subgroups. Finally, regarding WC, only differences in AtSE were noted, and according to BMI, there were differences in AtSE and GSE.

Table 5. Means and SD from post hoc tests in WC and BMI.

\begin{tabular}{|c|c|c|c|c|c|c|c|}
\hline \multirow{2}{*}{ Self-esteem } & \multirow{2}{*}{ Subgroup } & \multicolumn{3}{|c|}{ WC } & \multicolumn{3}{|c|}{ BMI } \\
\hline & & $\mathrm{n}$ & M & SD & $n$ & $\mathbf{M}$ & SD \\
\hline \multirow{5}{*}{ AcSE } & $a$ & 30 & 47.10 & 31.03 & 24 & 51.21 & 32.45 \\
\hline & $b$ & 185 & 54.19 & 28.30 & 201 & 54.96 & 28.92 \\
\hline & c & 65 & 58.32 & 26.83 & 47 & 61.06 & 23.19 \\
\hline & $d$ & 75 & 55.51 & 27.98 & 83 & 51.16 & 27.62 \\
\hline & Sig. differences & & & & & & \\
\hline \multirow{5}{*}{ AtSE } & $a$ & 30 & 53.93 & 27.63 & 24 & 60.50 & 25.97 \\
\hline & b & 185 & 60.59 & 23.89 & 201 & 58.76 & 25.16 \\
\hline & c & 65 & 53.12 & 26.97 & 47 & 55.85 & 25.55 \\
\hline & d & 75 & 47.49 & 29.93 & 83 & 47.65 & 29.22 \\
\hline & Sig. differences & & $b>d$ & & & $b>d$ & \\
\hline \multirow{5}{*}{ SSE } & $a$ & 30 & 50.60 & 27.29 & 24 & 50.50 & 29.35 \\
\hline & $b$ & 185 & 56.85 & 26.93 & 201 & 56.51 & 26.52 \\
\hline & $c$ & 65 & 56.54 & 28.60 & 47 & 62.77 & 28.09 \\
\hline & d & 75 & 55.12 & 29.69 & 83 & 52.10 & 29.76 \\
\hline & Sig. differences & & - & & & - & \\
\hline \multirow{5}{*}{ GSE } & $a$ & 30 & 47.70 & 28.49 & 24 & 51.13 & 30.51 \\
\hline & $b$ & 185 & 59.21 & 28.27 & 201 & 57.99 & 27.60 \\
\hline & c & 65 & 56.38 & 25.61 & 47 & 64.00 & 24.75 \\
\hline & $d$ & 75 & 53.52 & 28.17 & 83 & 50.29 & 28.52 \\
\hline & Sig. differences & & - & & & $c>d$ & \\
\hline
\end{tabular}

M: mean, SD: standard deviation, $n=$ sample size in subgroup.

WC: waist circumference; BMI: body mass index; ACSE: academic self-esteem; AtSE: athletic self-esteem; SSE: socio-affective and emotional self-esteem; GSE: global self-esteem.

a: underweight, b: normal weight, c: overweight, and d: obesity.

Significant differences found in ANOVA tests were examined through post hoc tests by using Tukey's HSD (Tables 4 and 5). Overall, significant results confirmed ANOVAs' results by showing that participants who reported higher scores in 20mSRT and SBJ also reported higher scores in AcSE, AtSE, SSE and GSE, in comparison with those whose HPF percentiles were lower (Tukey's HSD $p<.05$ ). Additionally, significant differences were also found between some consecutive subgroups. Regarding the $20 \mathrm{mSRT}$, significant differences were found between 'poor' and 'medium' subgroups in AcSE. About SBJ, 'very poor' participants scored lower than the 'poor' ones in SSE and GSE. Last, post hoc tests on body composition measures reported that normal-weight participants scored higher than obese participants on AtSE according to WC. Additionally, according to BMI, normal-weight children scored higher on AtSE than obese participants; also, overweight children showed higher GSE in comparison with the obese participants.

\section{DISCUSSION}

The present study aimed to determine the relationships between different components of HPF and selfesteem among children and young adolescents. CRF and lower-body musculoskeletal fitness were positively 
associated with self-esteem, whereas there were little significant correlations between body composition measures and self-esteem among children aged 9 to 13 years old.

Literature had already shown positive associations between CRF and physical health and quality of life in children (Borras et al., 2011). In the present study, positive associations were found between CRF and academic, athletic, social, and global self-esteem. Previous studies revealed these relationships between aerobic fitness and wellbeing in both children (Gálvez, Rodríguez, Rosa, García-Cantó, Pérez, et al., 2015) and adolescents (Jiménez-Moral et al., 2013). In relation to the magnitude of the associations, although they were generally low, the strongest association was found with AtSE, which is in line with previous evidence (García-Sánchez et al., 2013). In fact, post hoc analysis demonstrated that those children who showed very high CRF levels (> P80) significantly exceeded AtSE levels in comparison with the lower CRF groups. In the same way, it is shown that those participants with very low CRF also had an AtSE significantly more devalued even when they were compared to those with medium CRF ( $\geq$ P40 to $<P 60)$.

Musculoskeletal fitness was positively associated with self-esteem, which is consistent with previous works (Rodriguez et al., 2015; Smith et al., 2014). Focusing on the associations of SBJ and HS, it is remarkable that these associations only appear with the lower-body strength. This result is in line with findings of GarciaSánchez et al. (2013), who did not found relationship between HS and any dimensions of self-concept. Despite this, the findings of the present study showed that participants with greater muscular strength in the lower train showed higher scores in all dimensions of self-esteem, in comparison with those with lowest levels of muscular strength.

Results regarding body composition did not show associations as clear as the previous ones. There was only evidence of significant and negative correlations between BMI and AtSE, and WC and AtSE. This finding is consistent with the results of previous research that found a negative association between BMI and physical self-concept, as well as more specific dimensions of physical self-concept (Gálvez, Rodríguez, Rosa, GarcíaCantó, Pérez-Soto, et al., 2015; Gálvez, Rodríguez, Rosa, García-Cantó, Pérez, et al., 2015; Grao-Cruces et al., 2017). Literature has previously reported similar results in child population (Delgado-Floody et al., 2017; Flores et al., 2017).

In this sense, ANOVA and post hoc tests have shown that our results are in line with those studies that associate a higher weight status with less satisfaction with life (Padilla-Moledo, Castro-Piñero, et al., 2012) and with low self-esteem (Gálvez, Rodríguez, Rosa, García-Cantó, Pérez-Soto, et al., 2015; Hesketh et al., 2004 ) in childhood. However, it should be noted that we only found significant results in AtSE and global selfesteem. Specifically, it is noticeable that overweight participants, according to BMI, reported the highest scores in global self-esteem, although the differences are not statistically different from those with low and normal weight. This could be due to the early age of participants and the presence of males, as previous studies have demonstrated that these largely build their self-concept based on elements associated with fitness (Gálvez, Rodríguez, Rosa, García-Cantó, Pérez, et al., 2015). In addition to this, a possible CRF mediation between weight status and physical self-concept should not be ignored (Mitchell et al., 2012). Other studies have demonstrated that, even among samples of overweight and obese adolescents, better CRF levels are related to higher physical and global self-esteem (García-Sánchez et al., 2013; Grao-Cruces et al., 2017).

Regarding gender, little differences were found between males and females, except in HS, WC and AcSE, which were larger among females, and AtSC, which was higher among males. This fact is contrary to previous results that show boys reporting higher levels in HPF measures (Guillén and Ramírez, 2011; Rodriguez et 
al., 2015), although is consistent with those studies that found better results in males' self-esteem (Inglés et al., 2009). In this line, it should be noted that our sample was partially composed of preadolescents, who are in a life stage when self-concept is likely to decrease (Goñi et al., 2012). This could explain the lack of agreement with other studies.

This study is not without limitations. First, it is a cross-sectional study that does not allow to obtain any causeeffect relationship. Furthermore, the study of participants' HPF at these ages is difficult because of the lack of studies that establish appropriate reference values. However, the most updated normative values, based on a systematic review, have been used for most of the measured HPF components (Tomkinson et al., 2018), in order to avoid differing references. Finally, the sample size is not high enough to differentiate analyses by sex or weight status groups and ensure an adequate level of statistical power.

\section{CONCLUSIONS}

The results of the present study confirm a positive association between HPF components and self-esteem in children from 9 to 13 years old. In particular, CRF and lower-body musculoskeletal fitness showed small-tomedium positive associations with self-esteem. It is also suggested that a healthier weight status is associated with better athletic and global self-esteem. Considering the aforementioned benefits derived from having a good HPF, as well as the physical and psychological advantages from regular physical activity, our findings show evidence in favour of programmes focused on the improvement of HPF, especially, CRF and musculoskeletal fitness. These results may serve as a guideline in designing programmes aimed at improving self-esteem and physical health during school age.

\section{CONFLICT OF INTEREST}

The authors state that there are no conflicts of interest related to this study.

\section{ACKNOWLEDGEMENTS}

The researchers Álvaro Infantes-Paniagua and María Pilar León are supported by a grant from the Spanish Ministry of Education, Culture and Sport (FPU16/00082 and FPU15/00137, respectively).

The procedures described in this study complied with the Spanish legislation on human clinical research (Royal Decree 561/1993).

\section{REFERENCES}

Babic, M. J., Morgan, P. J., Plotnikoff, R. C., Lonsdale, C., White, R. L., \& Lubans, D. R. (2014). Physical activity and physical self-concept in youth: systematic review and meta-Analysis. Sports Med, 44, 1589-1601. https://doi.org/10.1007/s40279-014-0229-z

Borras, P. A., Vidal, J., Ponseti, X., Cantallops, J., \& Palou, P. (2011). Predictors of quality of life in children. J Human Sport Exercise, 6(4), 649-656. https://doi.org/10.4100/jhse.2011.64.08

Bouchard, C. E., Shephard, R. J., \& Stephens, T. E. (1994). Physical activity, fitness, and health: International proceedings and consensus statement. International Consensus Symposium on Physical Activity, Fitness, and Health, 2nd, May, 1992, Toronto, ON, Canada. Champaign, IL: Human Kinetics Publishers. https://doi.org/10.1002/ajhb.1310060517 
Cole, T. J., \& Lobstein, T. (2012). Extended international (IOTF) body mass index cut-offs for thinness, overweight and obesity. Pediatr Obes, 7(4), 284-294. https://doi.org/10.1111/j.2047$6310.2012 .00064 . x$

Collins, H., Booth, J. N., Duncan, A., Fawkner, S., \& Niven, A. (2019). The effect of resistance training interventions on 'the self' in youth: a systematic review and meta-analysis. Sports Med, 5(1), 29. https://doi.org/10.1186/s40798-019-0205-0

DeFina, L. F., Haskell, W. L., Willis, B. L., Barlow, C. E., Finley, C. E., Levine, B. D., \& Cooper, K. H. (2015). Physical activity versus cardiorespiratory fitness: Two (partly) distinct components of cardiovascular health? Prog Cardiovasc Dis, 57(4), 324-329. https://doi.org/10.1016/i.pcad.2014.09.008

Delgado-Floody, P., Caamaño-Navarrete, F., Jérez-Mayorga, D., Cofré-Lizama, A., Osorio-Poblete, A., Campos-Jara, C., ... Carcamo-Oyarzun, J. (2017). Obesidad, autoestima y condición física en escolares. Rev Fac Med, 65(1), 43-48. https://doi.org/10.15446/revfacmed.v65n1.57063

Fernández, C., Lorenzo, H., Vrotsou, K., Aresti, U., Rica, I., \& Sánchez, E. (2011). Estudio de crecimiento de Bilbao curvas y tablas de crecimiento (Estudio transversal). Fundación Faustino Orbegozo Eizaguirre.

Field, A. (2013). Discovering statistics using IBM SPSS statistics (4th edition). SAGE Publishing.

Flores, J. A. S., Fragante, J. F., Francisco, J. J. M., Dipasupila, J. P. P., Damaso, P. S. D., Gutierrez, L., ... Sison, R. S. (2017). The correlation between body mass index and self-esteem among children ages 9-12 years old in a public elementary school in Makati city, Philippines. Paediatrics Primary Care Physicians, 1(1), 13-17.

Gálvez, A., Rodríguez, P. L., Rosa, A., García-Cantó, E., Pérez-Soto, J. J., Marcos, L. T., \& López, P. T. (2015). Relación entre el estatus de peso corporal y el autoconcepto en escolares. Nutr Hosp, 31(2), 730-736. https://doi.org/10.3305/nh.2015.31.2.8467

Gálvez, A., Rodríguez, P. L., Rosa, A., García-Cantó, E., Pérez, J. J., Tárraga, P., \& Tárraga, L. (2015). Capacidad aeróbica, estado de peso y autoconcepto en escolares de primaria. Clín Inv Arteriosclerosis, 28(1), 1-8. https://doi.org/10.1016/j.arteri.2015.10.002

García-Sánchez, A., Burgueño-Menjibar, R., López-Blanco, D., \& Ortega, F. B. (2013). Physical fitness, adiposity and self-concept in adolescents. A pilot study. Rev Psicol Deporte, 22(2):453-461.

Garrido-Miguel, M., Cavero-Redondo, I., Álvarez-Bueno, C., Rodríguez-Artalejo, F., Moreno, L. A., Ruiz, J. R., ... Martínez-Vizcaíno, V. (2019). Prevalence and trends of overweight and obesity in European children from 1999 to 2016. JAMA Pediatr, e192430. https://doi.org/10.1001/jamapediatrics.2019.2430

Gerber, M., Endes, K., Brand, S., Herrmann, C., Colledge, F., Donath, L., ... Zahner, L. (2017). In 6- to 8-year-old children, cardiorespiratory fitness moderates the relationship between severity of life events and health-related quality of life. Qual Life Res, 26(3), 695-706. https://doi.org/10.1007/s11136-016-1472-6

Goñi, E., Fernández, A., \& Infante, G. (2012). El autoconcepto personal: diferencias asociadas a la edad y al sexo. Aula Abierta, 40(1), 39-50.

Grao-Cruces, A., Fernández-Martínez, A., \& Nuviala, A. (2017). Asociación entre condición física y autoconcepto físico en estudiantes españoles de 12-16 años. Rev Lat Am Psicol, 49(2), 128-136. https://doi.org/10.1016/j.rlp.2016.09.002

Guillén, F., \& Ramírez, M. (2011). Relación entre autoconcepto y condición física en alumnos del tercer ciclo de primaria. Rev Psicol Deporte, 20(1), 45-59.

Hesketh, K., Wake, M., \& Waters, E. (2004). Body mass index and parent-reported self-esteem in elementary school children: evidence for a causal relationship. Int J Obesity, 28(10), 1233-1237. https://doi.org/10.1038/sj.ijo.0802624 
Hill, A. J. (2017). Obesity in children and the 'myth of psychological maladjustment': Self-esteem in the spotlight. Current Obes Report, 6(1), 63-70. https://doi.org/10.1007/s13679-017-0246-y

Inglés, C. J., Pastor, Y., Torregrosa, M. S., Redondo, J., \& García-fernández, J. M. (2009). Diferencias en función del género y el curso académico en dimensiones del autoconcepto : estudio con una muestra de adolescentes españoles. Anuario Psicol, 40(2), 271-288.

Jiménez-Moral, J. A., Sánchez, M. L. Z., Molero, D., Pulido-Martos, M., \& Ruiz, J. R. (2013). Capacidad aeróbica, felicidad y satisfacción con la vida en adolescentes españoles. Rev Psicol Deporte, 22(2), 429-436.

Lang, J. J., Belanger, K., Poitras, V., Janssen, I., Tomkinson, G. R., \& Tremblay, M. S. (2018). Systematic review of the relationship between $20 \mathrm{~m}$ shuttle run performance and health indicators among children and youth. J Sci Med Sport, 21(4), 383-397. https://doi.org/10.1016/j.jsams.2017.08.002

Léger, L. A., Mercier, D., Gadoury, C., \& Lambert, J. (1988). The multistage 20 metre shuttle run test for aerobic fitness. J Sport Sci, 6(2), 93-101. https://doi.org/10.1080/02640418808729800

Mitchell, N. G., Moore, J. B., Bibeau, W. S., \& Rudasill, K. M. (2012). Cardiovascular fitness moderates the relations between estimates of obesity and physical self-perceptions in rural elementary school students. J Phys Act Health, 9(2), 288-294. https://doi.org/10.1123/jpah.9.2.288

Ortega, F. B., Artero, E. G., Ruiz, J. R., España-Romero, V., Jiménez-Pavón, D., Vicente-Rodríguez, G., ... \& Ciarapica, D. (2011). Physical fitness levels among European adolescents: the HELENA study. Brit J Sport Med, 45(1), 20-29. http://dx.doi.org/10.1136/bjsm.2009.062679

Ortega, F. B., Silventoinen, K., Tynelius, P., \& Rasmussen, F. (2012). Muscular strength in male adolescents and premature death: cohort study of one million participants. BMJ, 345, e7279-e7279. https://doi.org/10.1136/bmi.e7279

Padilla-Moledo, C., Castro-Piñero, J., Ortega, F. B., Mora, J., Marquez, S., Sjostrom, M., \& Ruiz, J. R. (2012). Positive health, cardiorespiratory fitness and fatness in children and adolescents. Eur J Public Health, 22(1), 52-56. https://doi.org/10.1093/eurpub/ckr005

Padilla-Moledo, C., Ruiz, J. R., Ortega, F. B., Mora, J., \& Castro-Piñero, J. (2012). Associations of muscular fitness with psychological positive health, health complaints, and health risk behaviors in Spanish children and adolescents. J Strength Cond Res, 26(1), 167-173. https://doi.org/10.1519/JSC.0b013e31821c2433

Ramos, R. (2008). Elaboración y validación de un cuestionario multimedia y multilingüe de evaluación de la autoestima. Universidad de Granada.

Rey, O., Vallier, J. M., Nicol, C., Mercier, C. S., \& Maïano, C. (2017). Effects of combined vigorous interval training program and diet on body composition, physical fitness, and physical self-perceptions among obese adolescent boys and girls. Pediatr Exerc Sci, 29(1), 73-83. https://doi.org/10.1123/pes.20160105

Rodriguez, P. L., Galvez, A., Garcia, E., Perez, J. J., Guillamon, A. R., Tarraga, L., \& Tarraga, P. J. (2015). Relationship between the self-concept and muscular strength in southern Spanish children. J Psychol Psychotherapy, 5(6). https://doi.org/10.4172/2161-0487.1000222

Rodriguez-Ayllon, M., Cadenas-Sanchez, C., Esteban-Cornejo, I., Migueles, J. H., Mora-Gonzalez, J., Henriksson, P., ... Ortega, F. B. (2018). Physical fitness and psychological health in overweight/obese children: A cross-sectional study from the ActiveBrains project. J Sci Med Sport, 21(2), 179-184. https://doi.org/10.1016/j.jsams.2017.09.019

Rosa, A., Garcia, P. E., \& Carrillo, J. (2019). Physical activity, physical fitness and self-concept in schoolchildren aged between 8 to 12 years old. Retos, 35, 236-241.

Rosa-Guillamón, A. (2019). Revisión bibliográfica de la relación entre condición física y autoconcepto. J Sport Health Res, 11(2), 117-128. 
Rosenberg, M. (1985). Self-concept and psychological well-being in adolescence. In R. L. Leary (Ed.), The development of the self (pp. 205-246). New York: Academic Press.

Ruiz, J. R., España, V., Castro, J., Artero, E. G., Ortega, F. B., Jiménez, D., ... Castillo, M. J. (2011). Manual de instrucciones. Batería ALPHA-Fitness: test de campo para la evaluación de la condición física relacionada con la salud en niños y adolescentes. Nutr Hosp, 26(6-Anexo), 1210-1214.

Shavelson, R. J., Hubner, J. J., \& Stanton, G. C. (1976). Self-concept: validation of construct interpretations. Rev Educ Res, 46(3), 407-441. https://doi.org/10.3102/00346543046003407

Smith, J. J., Eather, N., Morgan, P. J., Plotnikoff, R. C., Faigenbaum, A. D., \& Lubans, D. R. (2014). The health benefits of muscular fitness for children and adolescents: A systematic review and metaAnalysis. Sports Med, 44(9), 1209-1223. https://doi.org/10.1007/s40279-014-0196-4

Tomkinson, G. R., Carver, K. D., Atkinson, F., Daniell, N. D., Lewis, L. K., Fitzgerald, J. S., ... Ortega, F. B. (2018). European normative values for physical fitness in children and adolescents aged 9-17 years: results from 2.779.165 Eurofit performances representing 30 countries. Brit J Sport Med, 52(22), 1445-1456. https://doi.org/10.1136/bjsports-2017-098253

World Health Organization (2016). Informe de la comisión para acabar con la obesidad infantil. Ginebra. World Health Organization (2017). 10 datos sobre la obesidad. Retrieved from https://goo.gl/2bSMRU.

\section{@) $\odot \Theta \Theta$}

This work is licensed under a Attribution-NonCommercial-NoDerivatives 4.0 International (CC BY-NC-ND 4.0). 